\title{
HARMONIC FUNCTIONS ON HERMITIAN HYPERBOLIC SPACE
}

\author{
BY \\ ADAM KORÁNYI( $\left.{ }^{1}\right)$
}

Let $\mathscr{D}$ denote the complex unit ball

$$
\mathscr{D}=\left\{z=\left(z_{1}, \ldots, z_{n}\right)|| z \mid<1\right\}
$$

where $|z|$ is defined by $|z|^{2}=\sum\left|z_{k}\right|^{2}$. The Laplace-Beltrami operator for the Bergman metric of $\mathscr{D}$ is given $[4$, p. 117] by

$$
\Delta=\left(1-|z|^{2}\right)\left(\sum_{k} \frac{\partial^{2}}{\partial \bar{z}_{k} \partial z_{k}}-\sum_{k, l} \bar{z}_{k} z_{l} \frac{\partial^{2}}{\partial \bar{z}_{k} \partial z_{l}}\right) .
$$

We say that a function $F$ is harmonic in $\mathscr{D}$ if $\Delta F=0$, and we define the classes $\mathscr{H}^{p}(\mathscr{D})$ of harmonic functions in analogy with the classes $H^{p}(\mathscr{D})$ of holomorphic functions. The Poisson kernel corresponding to $\Delta$ is explicitly known [4]; since $\mathscr{D}$ is a symmetric space of rank one, $\mathscr{H}^{\infty}(\mathscr{D})$ is exactly the set of all Poisson integrals of $L^{\infty}(\mathscr{B}), \mathscr{B}$ denoting the boundary of $\mathscr{D}$ [3]. As we show by an easy reduction to the case of $p=\infty$, the same statement is true for every $p \geqq 1$ (with a slight modification if $p=1$ ).

Our main concern is the generalization of the classical Fatou theorem, and of its local version due to Privalov and Calderón ([1], [7]). For this purpose we define the notion of admissible convergence in $\S 3$. We show that in the case of $n=1$ admissible convergence coincides with nontangential convergence, while for $n>1$ it is stronger. It is a notion invariant under the group of holomorphic automorphisms of $\mathscr{D}$; nontangential convergence in the case $n>1$ is not. We prove Fatou's theorem for admissible convergence by some explicit estimates on the Poisson kernel and by using an extension of the Hardy-Littlewood Maximal Theorem due to Edwards and Hewitt [2]. It is perhaps worth mentioning that this is a new result even for holomorphic functions since previous investigations, being based on the euclidean Poisson integral, yielded only radial or nontangential convergence [1], [6].

The generalized Cayley transformation carries $\mathscr{D}$ onto a generalized halfplane $D$. In analogy with [5] one can again define the spaces of harmonic functions $\mathscr{H}^{p}(D)$. The results described above all have their analogues in this situation, and the proofs are parallel to those for $\mathscr{D}$. It should be noted, however, that these

Received by the editors May 29, 1967.

(1) Partially supported by NSF GP 6155. 
results are in general not equivalent since the Cayley transform does not carry $\mathscr{H}^{p}(\mathscr{D})$ onto $\mathscr{H}^{p}(D)$, except in the case of $p=\infty$. The local Fatou theorem, which involves only the case $p=\infty$, will be proved for the case of $D$, this case being easier to handle. Finally it will be indicated how all these results extend to products of domains of the type $\mathscr{D}$ or $D$.

I would like to express my thanks to E. M. Stein who, on my request, proved the version of the Maximal Theorem used in $\$ 4$ at a time before either he or I became aware of the article of Edwards and Hewitt [2].

1. Just as in [5] we denote by $\mu$ the normalized rotation-invariant measure on $\mathscr{B}$ (it is a constant multiple of the measure induced by the euclidean structure of $\left.C^{n}\right)$. We denote by $L^{p}(\mathscr{B})$ the usual $L^{p}$-space with respect to $\mu$ of complex-valued functions on $\mathscr{B}$. For a complex-valued function $F$ on $\mathscr{D}$ and $0<r<1$ we write $F_{r}(z)=F(r z)$. We define

$$
\mathscr{H}^{p}(\mathscr{D})=\left\{F: \mathscr{D} \rightarrow C \mid \Delta F=0, \sup _{0<r<1}\left\|F_{r}\right\|_{L^{p}(\mathscr{B})}<\infty\right\} .
$$

It is known [4], [5] that the Szegö and Poisson kernels of $\mathscr{D}$ are given by

$$
\begin{aligned}
& \mathscr{S}_{w}(z)=\mathscr{S}(z, w)=\frac{1}{(1-z \cdot \bar{w})^{n}} \quad(z, w \in \mathscr{D}), \\
& \mathscr{P}_{z}(u)=\mathscr{P}(u, z)=\frac{|\mathscr{S}(u, z)|^{2}}{\mathscr{S}(z, z)}=\frac{\left(1-|z|^{2}\right)^{n}}{|1-z \cdot \bar{u}|^{2 n}} \quad(z \in \mathscr{D}, u \in \mathscr{B}),
\end{aligned}
$$

where $z \cdot \bar{w}$ denotes $\sum z_{k} \bar{w}_{k}$.

Theorem 1. (i) $F$ is the Poisson integral of a function $f \in L^{p}(\mathscr{B})(1<p \leqq \infty)$ if and only if $F \in \mathscr{H}^{p}(\mathscr{D})$.

(ii) $F$ is the Poisson integral of a Baire measure on $\mathscr{B}$ if and only if $F \in \mathscr{H}^{1}(\mathscr{D})$.

(iii) $F$ is the Poisson integral of a function $f \in L^{1}(\mathscr{B})$ if and only if $F \in \mathscr{H}^{1}(\mathscr{D})$ and the family $\left\{F_{r} \mid 0<r<1\right\}$ is uniformly integrable.

Proof. The "only if" parts are immediate since the Poisson integral lifts as a convolution to the group $K=U(n)$ of holomorphic automorphisms fixing 0 (Theorem 4.7 in [5] and subsequent remarks).

The "if" part for $p=\infty$ is a consequence of Furstenberg's main theorem [3]. If $p<\infty$, let $\left\{\alpha_{n}\right\}$ be an approximate identity on the group $K$, i.e. a sequence of nonnegative $C^{\infty}$-functions of compact support each having integral 1 and converging to the delta measure based at the identity element of $K$. Defining $F_{n}(z)$ $=\int_{K} \alpha_{n}(g) F\left(g^{-1} z\right) d g, F_{n}$ is harmonic by the group-invariance of $\Delta$. For $0<r<1$ we have $F_{n, r}=\alpha_{n} * F_{r}$. Hence, $q$ denoting the conjugate exponent to $p$,

(1) $\left\|F_{n, r}\right\|_{\infty} \leqq\left\|\alpha_{n}\right\|_{q}\left\|F_{r}\right\|_{p} \leqq M\left\|\alpha_{n}\right\|_{q}$,

(2) $\left\|F_{n, r}\right\|_{p} \leqq\left\|\alpha_{n}\right\|_{1}\left\|F_{r}\right\|_{p} \leqq M$.

By (1), $F_{n}$ is the Poisson integral of some $f_{n} \in L^{\infty}(\mathscr{B})$. By (2) we have $\left\|f_{n}\right\|_{p} \leqq M$. It follows that the family $\left\{f_{n}\right\}$ is weakly compact in $L^{p}(\mathscr{B})$ in the case (i), in the 
space of Baire measures in case (ii) and in $L^{1}(\mathscr{B})$ in case (iii). Clearly $\lim F_{n}(z)$ $=F(z)$ for every fixed $z$, and the theorem follows.

2. For $0<\rho \leqq 1$ we define

$$
\mathscr{B}_{\rho}=\left\{\left.u \in \mathscr{B}|| \arg u_{1}\left|<\pi \rho, \sum_{2}^{n}\right| u_{k}\right|^{2}<\rho\right\} .
$$

We have $\mu\left(\mathscr{B}_{\rho}\right)=\rho^{n}$. For $\rho>1$ we define $\mathscr{B}_{\rho}=\mathscr{B}_{1}$. We denote $e=(1,0, \ldots, 0)$. For a function $f$ defined on $\mathscr{B}$ we define the maximal function $f^{*}$ by

$$
f^{*}(e)=\sup _{\rho>0} \frac{1}{\mu\left(\mathscr{B}_{\rho}\right)} \int_{\mathscr{B}_{\rho}}|f| d \mu
$$

and by $f^{*}(k e)=(f \circ k)^{*}(e)$ for $k \in K$. It is easy to see that $f^{*}$ is well defined on $\mathscr{B}$. Now define $K_{\rho}=\left\{k \in K \mid k e \in \mathscr{B}_{\rho}\right\}$. Then

$$
K_{\rho}=\left\{k=\left.\left(u_{i j}\right)|| \arg u_{11}\left|<\pi \rho, \sum_{2}^{n}\right| u_{i 1}\right|^{2}<\rho\right\}
$$

It is immediate that (i) the Haar measure of $K_{\rho}(\rho \leqq 1)$ is $\rho^{n}$, (ii) $\rho<\rho^{\prime}$ implies $K_{\rho} \subset K_{\rho^{\prime}}$, (iii) $\left(K_{\rho}\right)^{-1}=K_{\rho}$, (iv) there exists a number $m$ such that $K_{\rho} \mathscr{B}_{\rho} \subset \mathscr{B}_{m \rho}$. (For (iv) one can show e.g. by a simple computation that $K_{\rho} \mathscr{B}_{\rho} \subset \mathscr{B}_{6 \rho}$ for $\rho$ sufficiently small, and then infer the existence of $m$ by compactness.)

From these facts, making only some trivial modifications in the arguments of $[2, \S 2]$ one can prove the following version of the Maximal Theorem.

THEOREM 2. (i) For every $p>1$ there exists a constant $C_{p}$ such that $\left\|f^{*}\right\|_{p} \leqq C_{p}\|f\|_{p}$ for all $f \in L^{p}(\mathscr{B})$.

(ii) There exists a constant $C$ such that, for all $s>0$ and all $f \in L^{1}(\mathscr{B})$,

$$
\mu\left\{u \in \mathscr{B} \mid f^{*}(u)>s\right\} \leqq C \frac{\|f\|_{1}}{s}
$$

3. For every $0<\alpha<\infty$ we define the admissible domain at $u \in \mathscr{B}$,

$$
\mathscr{A}_{\alpha}(u)=\left\{z \in \mathscr{D} \mid \frac{|\mathscr{S}(u, z)|}{\mathscr{P}(u, z)}<\left(\frac{1+\alpha}{2}\right)^{n}\right\}
$$

For a function $F$ on $\mathscr{D}$ and a function $f$ on $\mathscr{B}$ we say that $F$ converges to $f$ admissibly (a.e.) if, for every $\alpha>0$,

$$
\lim _{z \rightarrow u: z \in \mathscr{A}_{\alpha}(u)} F(z)=f(u)
$$

for (almost) all $u \in \mathscr{B}$.

THEOREM 3. Admissible convergence is invariant under the group $G$ of holomorphic automorphisms of $\mathscr{D}$. 
Proof. Let $g \in G$. By [5, (3.4)],

$$
\frac{|\mathscr{S}(g z, g u)|}{\mathscr{P}(g z, g u)}=\frac{|\mathscr{S}(z, u)|}{\mathscr{P}(z, u)} \cdot \frac{\left|A_{g}(u)\right|}{\left|A_{g}(z)\right|}
$$

where $A_{g}$ is a nonvanishing holomorphic function on the closure of $\mathscr{D}$. By compactness, $\left|A_{g}(u)\right| \cdot\left|A_{g}(z)\right|^{-1}$ is between positive bounds, and the theorem follows.

In order to get a more geometrical description of admissible convergence we define, for $0<\alpha<\infty$,

$$
\Gamma_{\alpha}^{\prime}(e)=\left\{z \in \mathscr{D} \mid \frac{z}{|z|} \in \mathscr{B}_{\alpha(1-|z|)}\right\}
$$

and $\Gamma_{\alpha}^{\prime}(k e)=k \Gamma_{\alpha}^{\prime}(e)$ for $k \in K$. The following lemma then shows that in the definition of admissible convergence we can use $\Gamma_{\alpha}^{\prime}(u)$ instead of $\mathscr{A}_{\alpha}(u)$.

LEMMA 1. There exist constants $a, b, c, d$ such that $\mathscr{A}_{\alpha}(u) \subset \Gamma_{a \alpha+b}^{\prime}(u)$ and $\Gamma_{\alpha}^{\prime}(u)$ $\subset \mathscr{A}_{c \alpha+d}(u)$ for all $0<\alpha<\infty$ and all $u \in \mathscr{B}$.

For the proof one notices that $\mathscr{A}_{\alpha}(k e)=k \mathscr{A}_{\alpha}(e)(k \in K)$, and so it is enough to consider the case $u=e$. This case can be settled by a rather simple straightforward computation.

Next, we make the estimates necessary for Fatou's theorem.

LemMa 2. There exists a constant $C$ such that, for $0<r<1$,

$$
\begin{aligned}
\mathscr{P}_{r e}(u) & \leqq C /(1-r)^{n} & & (u \in \mathscr{B}), \\
& \leqq C(1-r)^{n} / \rho^{2 n} & & \left(u \in \mathscr{B}-\mathscr{B}_{\rho}\right) .
\end{aligned}
$$

The proof is a simple computation based on the explicit expression

$$
\mathscr{P}_{r e}(u)=\frac{\left(1-r^{2}\right)^{n}}{\left|1-r u_{1}\right|^{2 n}}
$$

LEMMA 3. For every $0<\alpha<\infty$ there exists a constant $C_{\alpha}$ such that, denoting by $F$ the Poisson integral of $f,|F(z)| \leqq C_{\alpha} f^{*}\left(u_{0}\right)$ for all $f \in L^{p}(\mathscr{B})(p \geqq 1), u_{0} \in \mathscr{B}$, $z \in \Gamma_{\alpha}^{\prime}\left(u_{0}\right)$.

Proof. By $K$-invariance it suffices to consider the case $u_{0}=e$. Let $z \in \Gamma_{\alpha}^{\prime}(e)$. Then, writing $|z|=r$, we have $z=r k e$ with some $k \in K_{\alpha(1-r)}$. By an obvious property of $\mathscr{P}, \mathscr{P}_{z}(u)=\mathscr{P}_{r k e}(u)=\mathscr{P}_{r e}\left(k^{-1} u\right)$. By remark (iv) in $\S 2, \rho \geqq \alpha(1-r)$ and $u \in \mathscr{B}-\mathscr{B}_{m \rho}$ now imply $k^{-1} u \in \mathscr{B}-\mathscr{B}_{\rho}$.

Let $\delta=m \alpha(1-r)$. We write $\mathscr{B}=\mathscr{B}_{\delta} \cup\left(\mathscr{B}_{2 \delta}-\mathscr{B}_{\delta}\right) \cup \ldots$. By the observation just made, $u \in \mathscr{B}_{2^{j+1} \delta}-\mathscr{B}_{2^{y} \delta}$ implies $k^{-1} u \in \mathscr{B}-\mathscr{B}_{2^{j} \alpha(1-r)}$; so Lemma 2 gives

$$
\begin{aligned}
|F(z)|= & \left|\int_{\mathscr{D}} f \mathscr{P}_{z} d \mu\right| \leqq \frac{C}{(1-r)^{n}} \int_{\mathscr{B}_{\delta}}|f| d \mu \\
& +\sum_{j=0}^{\infty} C \frac{(1-r)^{n}}{\left(2^{j} \alpha(1-r)\right)^{2 n}} \int_{\mathscr{B}_{2^{j}+1_{\delta}-\mathscr{D}_{2} j_{\delta}}|f| d \mu .}
\end{aligned}
$$


This is further increased by taking each integral in the sum over all of $\mathscr{B}_{2^{j+1}}$. The definition of $f^{*}$ now shows that

$$
|F(z)| \leqq C m^{n} \alpha^{n} f^{*}(e)+\left(C \frac{m^{n}}{\alpha^{n}} \sum_{j=0}^{\infty} \frac{1}{2^{n(j-1)}}\right) f^{*}(e)=C_{\alpha} f^{*}(e)
$$

The generalization of Fatou's theorem follows from Lemma 3 by a standard argument [7, Chapter XVII]:

Theorem 4. Let $f \in L^{p}(\mathscr{B})(p \geqq 1)$ and let $F$ be its Poisson integral. Then $F$ converges to f admissibly almost everywhere.

4. The generalized Cayley transform

$$
z_{1} \rightarrow i \frac{1+z_{1}}{1-z_{1}}, \quad z_{k} \rightarrow i \frac{z_{k}}{1-z_{1}} \quad(k \geqq 2)
$$

carries $\mathscr{D}$ onto

$$
D=\left\{z=\left.\left(z_{1}, \ldots, z_{n}\right)\left|h(z)=\operatorname{Im} z_{1}-\sum_{2}^{n}\right| z_{k}\right|^{2}>0\right\}
$$

The operator $\Delta$ is transformed into

$$
h(z)\left[4\left(\operatorname{Im} z_{1}\right) \frac{\partial^{2}}{\partial z_{1} \partial \bar{z}_{1}}+\sum_{2}^{n} \frac{\partial^{2}}{\partial z_{k} \partial \bar{z}_{k}}+2 i \sum_{2}^{n} \bar{z}_{k} \frac{\partial^{2}}{\partial z_{1} \partial \bar{z}_{k}}-2 i \sum_{2}^{n} z_{k} \frac{\partial^{2}}{\partial z_{k} \partial \bar{z}_{1}}\right] .
$$

We denote the boundary of $D$ in $C^{n}$ by $B$. As in [5] we have the measure $\beta$ on $B$ defined by

$$
\begin{aligned}
\int_{B} f(u) d \beta(u)= & \int f\left(\operatorname{Re} u_{1}+i \sum_{2}^{n}\left|u_{k}\right|^{2}, u_{2}, \ldots, u_{n}\right) \\
& \cdot d\left(\operatorname{Re} u_{1}\right) d\left(\operatorname{Re} u_{2}\right) d\left(\operatorname{Im} u_{2}\right) \cdots d\left(\operatorname{Im} u_{n}\right) .
\end{aligned}
$$

$L^{p}(B)$ is defined with the aid of $\beta$. For a function $F$ on $D$ and $t>0, F_{t}(z)=$ $F\left(z_{1}+i t, z_{2}, \ldots, z_{n}\right)$. We define

$$
\mathscr{H}^{p}(D)=\left\{F: D \rightarrow C \mid \Delta_{D} F=0, \sup _{t>0}\left\|F_{t}\right\|_{L^{p}(B)}<\infty\right\} .
$$

With $\rho(z, w)=i\left(\bar{w}_{1}-z_{1}\right)-2 \sum_{2}^{n} z_{k} \bar{w}_{k}$, we have $\rho(z, z)=2 h(z)$, and [5, Proposition 5.3]

$$
\begin{aligned}
& S(z, w)=\frac{\Gamma(n)}{2 \pi^{n}} \cdot \frac{1}{\rho(z, w)^{n}}, \\
& P(u, z)=\frac{|S(u, z)|^{2}}{S(z, z)}=\frac{\Gamma(n)}{2 \pi^{n}} \cdot \frac{\rho(z, z)^{n}}{|\rho(u, z)|^{2 n}}
\end{aligned}
$$

for the Szegö and Poisson kernel of $D$. We say that $F$ is the Poisson integral of a function $f$ on $B$ if $F(z)=\int f(u) P(u, z) d \beta(u)$.

By a repetition of the arguments used to prove Theorem 1 we can now prove the following. 
TheORem 5. (i) $F$ is the Poisson integral of a function $f \in L^{p}(B)(1<p \leqq \infty)$ if and only if $F \in \mathscr{H}^{p}(D)$.

(ii) $F$ is the Poisson integral of a finite Baire measure on $B$ if and only if $F \in \mathscr{H}^{1}(D)$.

(iii) $F$ is the Poisson integral of a function $f \in L^{1}(B)$ if and only if $F \in \mathscr{H}^{1}(D)$ and the family $\left\{F_{t} \mid t>0\right\}$ is uniformly integrable with respect to $\beta$.

This result, together with the formulas of $[5, \S 4]$ shows that the inverse Cayley transform carries $\mathscr{H}^{p}(D)$ into $\mathscr{H}^{p}(\mathscr{D})$, but not onto, unless $p=\infty$.

To generalize the Maximal Theorem, instead of $K$ we consider the group $N$ of elements $(a, c)=\left(a, c_{2}, \ldots, c_{n}\right) \in \boldsymbol{R} \times \boldsymbol{C}^{n-1}$ acting on $D$ by the holomorphic autmorphisms

$$
\begin{aligned}
&(a, c): z_{1} \mapsto z_{1}+a+2 i \sum_{2}^{n} z_{k} \bar{c}_{k}+i \sum_{2}^{n}\left|c_{k}\right|^{2}, \\
&: z_{k} \mapsto z_{k}+c_{k} \quad(k \geqq 2) .
\end{aligned}
$$

$N$ leaves $\rho(z, w)$, hence also $h(z), S(z, w)$ and $P(u, z)$ invariant. We define

For $u \in B$ we define

$$
|(a, c)|=\operatorname{Max}\left\{|a|, \sum_{2}^{n}\left|c_{k}\right|^{2}\right\}
$$

$$
\|u\|=\operatorname{Max}\left\{\left|\operatorname{Re} u_{1}\right|, \sum_{2}^{n}\left|u_{k}\right|^{2}\right\}
$$

and for $\rho>0$ we define $B_{\rho}=\{u \in B \mid\|u\|<\rho\}, N_{\rho}=\{g \in N|| g \mid<\rho\}$. Clearly we have $N_{\rho}=\left\{g \in N \mid g \cdot 0 \in B_{\rho}\right\}$. If $g=(a, c)$, then $g^{-1}=(-a,-c)$; this shows that $N_{\rho}^{-1}=N_{\rho}$. Given two elements, $g=(a, c), g^{\prime}=\left(a^{\prime}, c^{\prime}\right)$ one checks by an easy computation that

$$
g g^{\prime}=\left(a+a^{\prime}-2 \operatorname{Im} \sum_{2}^{n} c_{k}^{\prime} \bar{c}_{k}, c+c^{\prime}\right)
$$

From this it follows that $\left|g g^{\prime}\right| \leqq 2\left(|g|+\left|g^{\prime}\right|\right)$, and this inequality implies that $N_{\rho} B_{\rho} \subset N_{4 \rho}$ for all $\rho>0$.

We define, for $f \in L^{p}(B)(p \geqq 1)$

$$
f^{*}(0)=\sup _{\rho>0} \frac{1}{\beta\left(B_{\rho}\right)} \int_{B_{\rho}}|f| d \beta
$$

and $f^{*}(g \cdot 0)=(f \circ g)^{*}(0)(g \in N)$. The Maximal Theorem can now be proved for $f^{*}$ by the methods of [2].

For $\alpha>0$ we define the admissible domain at $u \in B$ by

$$
A_{\alpha}(u)=\left\{z \in D \mid \frac{|S(u, z)|}{P(u, z)}<\left(\frac{1+\alpha}{2}\right)^{n}\right\}
$$

and we have a corresponding notion of admissible convergence.

THEOREM 6. Admissible convergence on $D$ is a notion invariant under the group of those holomorphic automorphisms of $D$ which have a continuous extension to $B$. 
Proof. The group in question is isomorphic under the Cayley transform with the subgroup of $G$ fixing the point $e$ on the boundary of $\mathscr{D}$. It is known (and easy to check) that it is generated by $N$ and by the maps $z_{1} \mapsto s z_{1}, z_{k} \mapsto s^{1 / 2} z_{k}$ $(k=2, \ldots, n), s>0$. One sees that for every fixed $\alpha$ these mappings only permute the corresponding domains $A_{\alpha}(u)$, whence the assertion follows.

The analogues of the domains $\Gamma_{\alpha}^{\prime}(u)$ are defined by

$$
\Gamma_{\alpha}(0)=\{z \in D \mid\|z-i h(z) e\|<\alpha h(z)\}
$$

and $\Gamma_{\alpha}(g \cdot 0)=g \Gamma_{\alpha}(0)$. Equivalently one can write

$$
\Gamma_{\alpha}(g \cdot 0)=\left\{g^{\prime} \cdot(\text { ite })|| g^{-1} g^{\prime} \mid<\alpha t\right\} .
$$

A straightforward computation now gives

Lemma 4. For every $\alpha>0, u \in B$ we have $A_{\alpha}(u) \subset \Gamma_{\alpha+1}(u)$ and $\Gamma_{\alpha}(u) \subset A_{2 \alpha}(u)$.

This shows that admissible convergence can again be equivalently redefined by using the $\Gamma_{\alpha}(u)$ instead of the $A_{\alpha}(u)$.

The basic estimate on the Poisson kernel is now

$$
\begin{aligned}
P(u, i t e) & \leqq c / t^{n} & & (u \in B), \\
& \leqq c t^{n} / \rho^{2 n} & & (\|u\| \geqq \rho),
\end{aligned}
$$

as one sees immediately from the explicit formula

$$
P(u, \text { ite })=c \frac{t^{n}}{\left(\left(\operatorname{Re} u_{1}\right)^{2}+\left(t+\sum_{2}^{n}\left|u_{k}\right|^{2}\right)^{2}\right)^{n}} .
$$

Proceeding from here in the same way as in the case of $\mathscr{D}$ we obtain the following version of Fatou's theorem.

TheOrem 7. Let $f \in L^{p}(B)(p \geqq 1)$ and let $F$ be its Poisson integral. Then $F$ converges to $f$ admissibly almost everywhere.

In concluding this section let us note that, although we defined the notions of admissible convergence in $\mathscr{D}$ and $D$ independently, they can easily be tied up with each other. In fact one can check that, at least in a neighborhood of 0 , there is a relation of the type of Lemmas 1 and 4 between $A_{\alpha}(0)$ and the Cayley transform of $\mathscr{A}_{\alpha}(-e)$.

5. In this section we follow closely the argument of Calderón [1], [7]. For $\alpha>0$ and $h>0$ we define the truncated domains

$$
\Gamma_{\alpha}^{h}(g \cdot 0)=\left\{g^{\prime} \cdot \text { ite }|0<t<h,| g^{-1} g^{\prime} \mid<\alpha t\right\} .
$$

LEMMA 5. Let $E \subset B$ and let $u_{0}$ be a point of density of $E$ with respect to the family of sets $\left\{g B_{\rho} \mid g \in N, \rho>0\right\}$. Then, given any $\alpha>0, h>0, \alpha_{0}>0$, there exists $h_{0}>0$ such that

$$
\Gamma_{\alpha_{0}}^{h_{0}}\left(u_{0}\right) \subset \bigcup_{u \in E} \Gamma_{\alpha}^{h}(u)
$$


Proof. We write $u_{0}=g_{0} \cdot 0\left(g_{0} \in N\right)$. For $u_{1}=g_{1} \cdot 0 \in B$ we define

$$
D_{g_{1}}=g_{1} B_{\alpha_{0}^{-1} \alpha\left|g_{0}^{-1} g_{1}\right|} \text {. }
$$

Now, for any $g \cdot 0(g \in N), g \cdot 0 \in D_{g_{1}}$ implies

$$
\left|g_{0}^{-1} g\right|=\left|\left(g_{0}^{-1} g_{1}\right)\left(g_{1}^{-1} g\right)\right| \leqq 2\left(\left|g_{0}^{-1} g_{1}\right|+\left|g_{1}^{-1} g\right|\right)<2\left(1+\frac{\alpha}{\alpha_{0}}\right)\left|g_{0}^{-1} g_{1}\right|
$$

This shows that the set $D^{\prime}=g_{0} B_{2\left(1+\alpha_{0}^{-1} \alpha\right)\left|g_{0}^{-1} g_{1}\right|}$ contains $D_{g_{1}}$. We have

$$
\beta\left(D_{g_{1}}\right) / \beta\left(D^{\prime}\right)=\alpha^{n} / 2^{n}\left(\alpha_{0}+\alpha\right)^{n} .
$$

Since $u_{0}$ is a point of density, it follows that there exists $c>0$ such that $\left|g_{0}^{-1} g_{1}\right|<c$ implies $D_{g_{1}} \cap E \neq \varnothing$.

We show that for any $h_{0}>0$ such that $h_{0}<h, c / \alpha_{0}$ has the required property. Let $z \in \Gamma_{\alpha_{0}}^{h_{0}}\left(u_{0}\right)$. Then $z=g_{1} \cdot i t_{1} e$ with $0<t_{1}<h,\left|g_{0}^{-1} g_{1}\right|<\alpha_{0} t_{1}$. By the choice of $h_{0}$, $\left|g_{0}^{-1} g_{1}\right|<c$, hence $D_{g_{1}} \cap E \neq \varnothing$. Let $u=g \cdot 0 \in D_{g_{1}} \cap E$. Then

$$
\left|g^{-1} g_{1}\right|=\left|g_{1}^{-1} g\right|<\left(\alpha / \alpha_{0}\right)\left|g_{0}^{-1} g_{1}\right|<\alpha t_{1},
$$

i.e., $z \in \Gamma_{\alpha}^{h}(u)$, finishing the proof.

THEOREM 8. Let $F$ be a harmonic function on $D$. Let $E \subset B$ be measurable and suppose that for every $u \in E$ there exist $\alpha>0, h>0$ such that $F$ is bounded in $\Gamma_{\alpha}^{h}(u)$. Then, at almost every point of $E, F$ converges admissibly to a finite boundary value.

Proof. First we note that it may be assumed that $\alpha$ and $h$ are the same for each $u \in E$, that $|F| \leqq M$ uniformly in every $\Gamma_{\alpha}^{h}(u)$, and that $E$ is bounded and closed. In fact, defining

$$
E_{j k l}=\left\{u \in E|| F \mid \leqq j \text { in } \Gamma_{1 / l}^{1 / k}(u)\right\},
$$

for any $\varepsilon>0$ there exists $\Omega$ such that, writing $E_{1}=\bigcup_{j, k, l \leqq \Omega} E_{j k l}, \beta\left(E-E_{1}\right)<\varepsilon$. Since $F$ is continuous, the closure of $E_{1}$ has the same property as $E_{1}$, and our statement follows.

Now let $\Gamma=\bigcup_{u \in E} \Gamma_{\alpha}^{h}(u)$. We will show that $F=p+r$ where $p \in \mathscr{H}^{\infty}(D)$ (so it converges admissibly a.e. on $B$ ), and $|r|$ is majorized in $\Gamma$ by a positive harmonic function $v$ which converges admissibly to 0 a.e. on $E$. Lemma 5 will then imply that $r$ also converges admissibly to 0 a.e. on $E$.

For this purpose we define the functions $f_{n}$ on $B$ by

$$
\begin{aligned}
f_{n}(u) & =F_{1 / n}(u) & & \text { if } u+i(e / n) \in \Gamma \\
& =0 & & \text { otherwise }
\end{aligned}
$$

and let $p_{n}$ be the Poisson integral of $f_{n} \cdot\left|p_{n}\right| \leqq M$ for each $n$; by weak compactness a subsequence of $\left\{p_{n}\right\}$ converges to some function $p \in \mathscr{H}^{\infty}(D)$.

Writing $r_{n}=F_{1 / n}-p_{n}$, the subsequence of $\left\{r_{n}\right\}$ corresponding to the above converges to a harmonic function $r$ such that $F=p+r$. Note that each $r_{n}$ is continuous on $\bar{\Gamma}, 0$ on $E$, and $\left|r_{n}\right| \leqq 2 M$ on $\Gamma$. 
Define $v$ by $v(z)=(2 M / h) h(z)+C w(z)$, where $w$ is the Poisson integral of $E^{\prime}$ (the complement of $E$ in $B$ ), and $C$ is a constant to be determined later. It is easy to see that $h(z)$, and hence $v$, is harmonic. To show $|r| \leqq v$ in $\Gamma$ it is enough to show $\left|r_{n}\right| \leqq v$ for each $n$. By the maximum principle it is enough to show this on $\partial \Gamma$, the boundary of $\Gamma$.

So let $z \in \partial \Gamma$. We distinguish three cases. (i) If $h(z)=0$, i.e. $z \in E$, then $r_{n}(z)=0$, and $\left|r_{n}(z)\right| \leqq v(z)$ is trivially true. (ii) If $h(z)=h$, then by $\left|r_{n}(z)\right| \leqq 2 M$ and $w(z) \geqq 0$, $\left|r_{n}(z)\right| \leqq v(z)$ for any choice of $C \geqq 0$. (iii) If $0<h(z)<h$, then $z \notin \Gamma_{\alpha}^{h}(u)$ for all $u \in E$, by definition of $\Gamma$. Writing $z=g^{\prime} \cdot$ ite, this means that $\left|g^{-1} g^{\prime}\right| \geqq \alpha t$ for all $g \in N$ such that $g \cdot 0 \in E$, i.e. $g^{\prime} B_{\alpha t} \subset E^{\prime}$. By some obvious changes of variables we have now

$$
\begin{aligned}
w(z) & =\int_{E^{\prime}} P(u, z) d \beta(u) \geqq \int_{g^{\prime} B_{\alpha t}} P(u, z) d \beta(u) \\
& =\int_{B_{\alpha t}} P(u, \text { ite }) d \beta(u)=\int_{B_{\alpha}} P(u, i e) d \beta(u)=C_{\alpha} .
\end{aligned}
$$

Hence, choosing $C=2 M / C_{\alpha}$, we again have $\left|r_{n}(z)\right| \leqq v(z)$, and the proof is finished.

6. For $j=1, \ldots, k$ let $\mathscr{D}_{j}$ be a complex ball in some $C^{n_{j}}$, and let $\mathscr{D}=\mathscr{D}_{1} \times \cdots \times \mathscr{D}_{k}$. It is well known that the Szegö kernel of $\mathscr{D}$ is $\mathscr{S}(z, w)=\Pi \mathscr{S}_{j}\left(z^{j}, w^{j}\right)$, and a similar relation holds for the Poisson kernel. Noticing that, by the formulas of $\S 1$, $\left|\mathscr{S}_{j}\left(u^{j}, z_{j}\right)\right| \cdot \mathscr{P}_{j}\left(u^{j}, z^{j}\right)^{-1}$ has a positive lower bound, it follows that $|\mathscr{S}(u, z)|$ $\cdot \mathscr{P}(u, z)^{-1}$ is bounded from above if and only if $\left|\mathscr{S}_{j}\left(u^{j}, z^{j}\right)\right| \cdot \mathscr{P}_{j}\left(u^{j}, z^{j}\right)^{-1}$ is bounded from above for each $j$. Therefore it is reasonable to define (unrestricted) admissible convergence for $\mathscr{D}$ in formally the same way as in $\S 3$.

Restricted admissible convergence is defined by adding the condition that as $z=\left(z^{1}, \ldots, z^{k}\right) \rightarrow u$, besides $z$ staying in an admissible domain, $1-\left|z^{r}\right|<M\left(1-\left|z^{s}\right|\right)$ should be satisfied for some $M$ and all $r, s=1, \ldots, k$. With these definitions, by the argument of [7, Chapter XVII] we obtain the following.

TheOREM 9. If $f \in L^{p}\left(\mathscr{B}_{1} \times \cdots \times \mathscr{B}_{k}\right)(p>1)$ and $F$ is its Poisson integral, then $F$ converges to fadmissibly a.e. If $\in L^{1}\left(\mathscr{B}_{1} \times \cdots \times \mathscr{B}_{k}\right)$, then $F$ converges to fadmissibly and restrictedly a.e.

Of course, a similar theorem is true for products of domains of type $D$, or even for a mixture of the two types.

Our proof of Theorem 8 can also be extended to the case of products of domains $D$. The additional arguments one has to make are exactly the same as in the case of products of halfplanes and are explicitly pointed out in [7, Chapter XVII].

\section{REFERENCES}

1. A. P. Calderón, On the behaviour of harmonic functions at the boundary, Trans. Amer. Math. Soc. 68 (1950), 47-54.

2. R. E. Edwards and E. Hewitt, Pointwise limits of convolution operators, Acta Math. 113 (1965), 181-218. 
3. H. Furstenberg, A Poisson formula for semi-simple Lie groups, Ann. of Math. 77 (1963), 335-386.

4. L. K. Hua, Harmonic analysis of functions of several complex variables in the classical domains, Transl. Math. Monographs, Vol. 6, Amer. Math. Soc., Providence, R. I., 1963.

5. A. Korányi, The Poisson integral for generalized halfplanes and bounded symmetric domains, Ann. of Math. 82 (1965), 332-350.

6. H. E. Rauch, Harmonic and analytic functions of several variables and the maximal theorem of Hardy and Littlewood, Canad. J. Math. 8 (1956), 171-183.

7. A. Zygmund, Trigonometric series, Cambridge Univ. Press, New York, 1959.

Belfer Graduate School, Yeshiva University, NeW YoRK, NeW YoRK 